Article

\title{
Oxide Self-Flux in Optical Floating Zone Crystal Growth of Nickel Niobate $\left(\mathrm{NiNb}_{2} \mathrm{O}_{6}\right)$
}

\author{
Timothy J.S. Munsie ${ }^{1}$, Anna Millington ${ }^{1}$, Graeme M. Luke ${ }^{1,2,3}$ and Hanna A. Dabkowska ${ }^{2, *}$ \\ 1 Department of Physics and Astronomy, McMaster University \\ 2 Brockhouse Institute for Materials Research, McMaster University \\ 3 Canadian Institute For Advanced Research, Toronto, Canada \\ * Correspondence: dabkoh@mcmaster.ca; Tel.: +1-905-525-9140 x27092
}

\begin{abstract}
Growing crystals of nickel niobate $\left(\mathrm{NiNb}_{2} \mathrm{O}_{6}\right)$, we noticed that changing growth conditions allowed our material to enter different areas of the phase diagram. We also found that excess material accumulated within and above the liquid zone. Analysis showed that this was an excess of $\mathrm{NiO}$. Changing the ratio of the constituent oxides - an excess of $\sim 4 \%$ of either $\mathrm{NiO}$ or $\mathrm{Nb}_{2} \mathrm{O}_{5}$ gave us the opportunity to investigate changes in zone stability, melting temperature and quality of the resulting crystal. We found that a small excess of nickel oxide decreases the melting temperature significantly, and created the best pseudo-rutile $\mathrm{NiNb}_{2} \mathrm{O}_{6}$ crystal studied (Pbcn, space group 60), while higher amounts of niobium oxide allowed us to stabilize the $\mathrm{NiNb}_{2} \mathrm{O}_{6}$ columbite phase $\left(\mathrm{P}_{2} / \mathrm{n}\right.$, space group 80$)$. This research reinforces the idea that self-flux as a travelling solvent can significantly impact crystal growth parameters and quality.
\end{abstract}

Keywords: optical floating zone; self flux; crystal growth; oxide growth

PACS: $81.10 . \mathrm{Fq}$

\section{Introduction}

The optical floating zone (OFZ) crystal growth method creates an opportunity to grow crystals of congruently and incongruently melting oxides [1]. Whereas to obtain crystals of congruently melting materials the grower has to well understand the interplay between melting temperatures, pulling speeds and growth atmospheres, the growth of incongruently melting oxides is much more demanding. Understanding of the applicable phase diagram allows for finding the appropriate "flux", which not only helps to grow the proper phase but also to stabilize it in post growth heat treatment.

Cobalt niobate $\left(\mathrm{CoNb}_{2} \mathrm{O}_{6}\right)$, which has previously been grown and studied by our group [2], has been identified as a material with a quantum critical point [3-6], a topic that has been of significant interest recently in the physics community [7-15]. QCPs are the boundaries of phase transitions that are not driven by temperature changes. Cobalt niobate consists of ferromagnetic spin $-\frac{1}{2}$ chains along the b-axis, with neighbouring chains weakly coupled antiferromagnetically due to isolation by non-magnetic niobium atoms [16]. This gives rise to an effective 1D Ising system. Testing the physical realization of the 1D Ising system is useful in the determination of how well the theoretical model matches nature.

Following the previous research done on the transverse field Ising spin model, we decided to extend our investigation of this system to the spin- 1 analogue, nickel niobate $\left(\mathrm{NiNb}_{2} \mathrm{O}_{6}\right)$. Both cobalt niobate and nickel niobate have previously been reported to form the columbite structure [17-19], 
space group $60(\mathrm{Pbcn})$, and reports on successful growths via the optical floating zone have been presented in [20], although no experimental details were given. The $\mathrm{NiNb}_{2} \mathrm{O}_{6}$ material has not been well explored due to the complicated phase diagram [21] and having high quality crystals of it would allow us to compare and contrast a potential spin- 1 magnetic system against a similar spin $-\frac{1}{2}$ system. Specifically this would let us examine the regime under which the spin- $\frac{1}{2}$ system undergoes a quantum phase transition, which is not likely in a spin-1 system. Some results on single crystals of columbite $\mathrm{NiNb}_{2} \mathrm{O}_{6}$ grown via flux methods are available for heat capacity and magnetometry [22,23].

In this paper, we report the growth of small single crystals of nickel niobate, up to a size of $\sim 5$ $\mathrm{mm} \times 3 \mathrm{~mm} \times 1 \mathrm{~mm}$. We did not succeed in obtaining one single crystalline boule, which was the case with $\mathrm{CoNb}_{2} \mathrm{O}_{6}$ [2], but after 16 growths, generally forming an external, polycrystalline shell of columbite-structure nickel niobate and various internal phases, we can report creating good quality single crystals of both the columbite and pseudo-rutile phases of $\mathrm{NiNb}_{2} \mathrm{O}_{6}$. The latter phase was a previously unreported polymorph of nickel niobate $\left(\beta-\mathrm{NiNb}_{2} \mathrm{O}_{6}\right)$ [24]. This particular structure was of additional interest due to the fact the space group that it was in, $\mathrm{P}_{2} / \mathrm{n}$ (space group 86), has not been reported before in the family of $\mathrm{AB}_{2} \mathrm{O}_{6}$ materials [25].

We have recently reported the basic structure and magnetic response data for $\beta-\mathrm{NiNb}_{2} \mathrm{O}_{6}$ [24] in space group 86 and here we report the methodology behind the synthesis of the material and various attempts made in an effort to stabilize $\beta-\mathrm{NiNb}_{2} \mathrm{O}_{6}$ as well as the columbite phase of $\mathrm{NiNb}_{2} \mathrm{O}_{6}$ via the optical floating zone growth technique. This paper details the effects of changing the growth atmosphere, the growth speed and the material initial stoichiometry on the resultant grown boule.

\section{Results}

The first two growth attempts were done in a Crystal Systems optical floating zone image furnace near the maximum power settings of the system ( $4 \times 300 \mathrm{~W}$ lamps). Remaining growths were performed in a NEC OFZ image furnace with higher wattage $(2 \times 1 \mathrm{~kW})$ lamps.

Initially, the growths were done in reducing conditions, required to stabilize the $\mathrm{NiNb}_{2} \mathrm{O}_{6}$ phase [21], using a commercially mixed gas of $90 \%$ argon and $10 \%$ hydrogen in an attempt to obtain the columbite structure. At the beginning the growths were done with a significant number of manual adjustments. Gas pressure was modified throughout the growth in an attempt to reach more stable conditions. After growth 4, all growths except 13, which was performed in air, were done in a $100 \%$ oxygen gas $\left(\mathrm{O}_{2}\right)$ environment at $200 \mathrm{kPa}$ overpressure up to growth $8,40 \mathrm{kPa}$ overpressure for growth 9 and with no overpressure for all remaining growths.

On the NEC system we were able to stabilize the growth at the rate of about $4 \mathrm{~mm} / \mathrm{hr}$. Although ultimately the zone spontaneously lost stability and began to widen to the point that the growth was unsustainable. A subsequent attempt at a slightly increased speed was also unstable, leading to the conclusion that this material grows best at $3 \mathrm{~mm} / \mathrm{hr}$ in the reducing atmosphere.

Because the material is not congruently melting, evidenced by the residual material left at the end of the growth, we decided to pre-melt it in an oxygen environment, as we wanted to densify the rod and make it uniform. Based on the stability exhibited during the pre-melt we continued growing in oxygen for future attempts. Growth of the pre-melted boule at a speed slower than $3 \mathrm{~mm} / \mathrm{hr}$, consistent with successful speeds used for growth of cobalt niobate, was unsuccessful.

The following growth was done at $10 \mathrm{~mm} / \mathrm{hr}$, which resulted in a boule of material with one dominant orientation. This was then grown at the most stable observed speed, which was about 5 $\mathrm{mm} / \mathrm{hr}$. Post-analysis showed that the tip was overheated and that a concave crystallization front was formed. Based on this we decided to reduce the oxygen overpressure for the next growths as stated above.

With $1.4 \%$ extra nickel oxide $(\mathrm{NiO})$ added via a flux pellet, we again pre-melted the rod. This resulted in a crystal with a facet. Within the boule there was a mixture of polycrystalline columbite, a black phase consisting of a mixture of columbite and the pseudo-rutile phase, as well as a few green single crystals, approximately $5 \mathrm{~mm} \times 3 \mathrm{~mm} \times 1 \mathrm{~mm}$, that were pure examples of $\beta-\mathrm{NiNb}_{2} \mathrm{O}_{6}$. A slice 


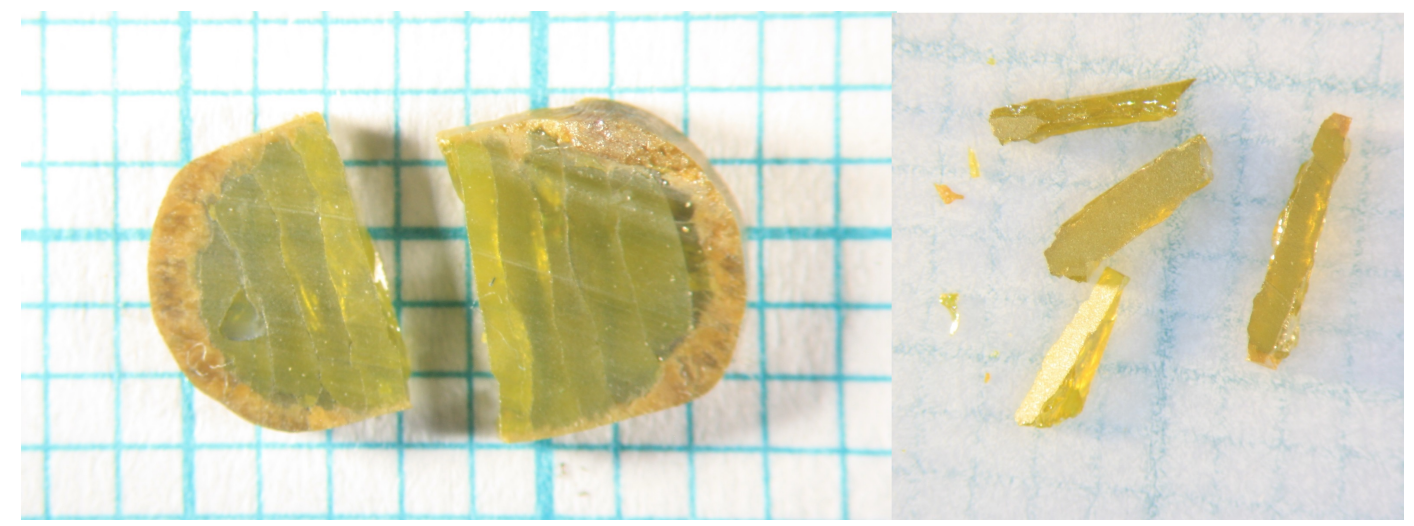

Figure 1. As grown crystal from growth 10, using a nickel oxide flux. The outer core is yellow, polycrystalline columbite. The green crystals in the centre are $\beta-\mathrm{NiNb}_{2} \mathrm{O}_{6}$, in single crystalline form as verified by both Laue and single-crystal x-ray scattering. The right hand panel shows the crystals separated after applying light, mechanical pressure with a scalpel between the ceramic and crystal portions, as well as along the fractures within the crystal itself. Lattice parameters for this sample found via x-ray scattering are a $=6.6893(3)$ Åand c $=9.0956(11) \AA$. Full refinement details have been published in reference [24].

of this material is shown in figure 1 . The powder $\mathrm{x}$-ray pattern is shown in figure 6 with the reference pattern for the tri-rutile $\left(\mathrm{P}_{2} / \mathrm{mnm}\right) \mathrm{NiNb}_{2} \mathrm{O}_{6}$ pattern from reference [19]. The boule was quenched according to the phase diagram [21] to stabilize the as grown phase.

Attempts made with higher concentrations of nickel oxide were unsuccessful at producing more of the green crystals, as can be seen in figure 2. We did, however, manage to stabilize black crystals, which were crystallographically identified as the same material but with a niobium deficiency. At the highest concentration, the additional material drove the resultant crystal back into the columbite area of the phase diagram. Both an amber and black columbite niobate (growth 16) are shown in figure 4.

The growth of the material with an excess of niobium oxide produced boules of both the black $\beta-\mathrm{NiNb}_{2} \mathrm{O}_{6}$ (growth 15) and the black columbite (growth 14), seen in figure 3 with the primary difference being the growth speed. A powder x-ray comparison can be seen in figure 6 for growth 15 and in figure 5 for growth 14 . In figure 5 the pattern is compared with one of the yellow polycrystalline pieces from the surface (in this case, from growth 12) as well as with the expected pattern for the columbite form from the database [17]. The obtained crystals were quenched, and this may be the reason for mechanical fracturing into plates. For $\beta-\mathrm{NiNb}_{2} \mathrm{O}_{6}$, the faces of the plates were perpendicular to the growth direction and oriented along [110]. These can be seen in figure 3 .

\section{Discussion}

$\beta-\mathrm{NiNb}_{2} \mathrm{O}_{6}$ was most stable when grown at higher speeds and with an excess of niobium oxide present as a flux. At slower speeds the system does form this phase, and the outcome is polycrystalline material, or small single crystals (mixed black and dark green) no larger than $1 \mathrm{~mm} x$ $1 \mathrm{~mm} \times 1 \mathrm{~mm}$. Additionally, there is almost always an outer shell of yellow polycrystalline columbite. The thickness of the shell depended on the atmosphere in which it is grown. Increasing the oxygen content as well as an excess of niobium oxide tended to make the outer shell thinner.

Attempts to stabilize the material as a single crystal by growing slower does not visually create the same material. Based on x-ray analysis, we get a niobium deficient version, with vacancies on the niobium sites. Under a stereoscope, the material appears black and opaque, but thin pieces under light are transparent and dark green in colour. A SQUID magnetometry assessment of the black and the green material shows that the magnetic response does not change with slight deficiencies of niobium. A portion of these SQUID analyses appears in reference [24]. This is not an unsurprising 


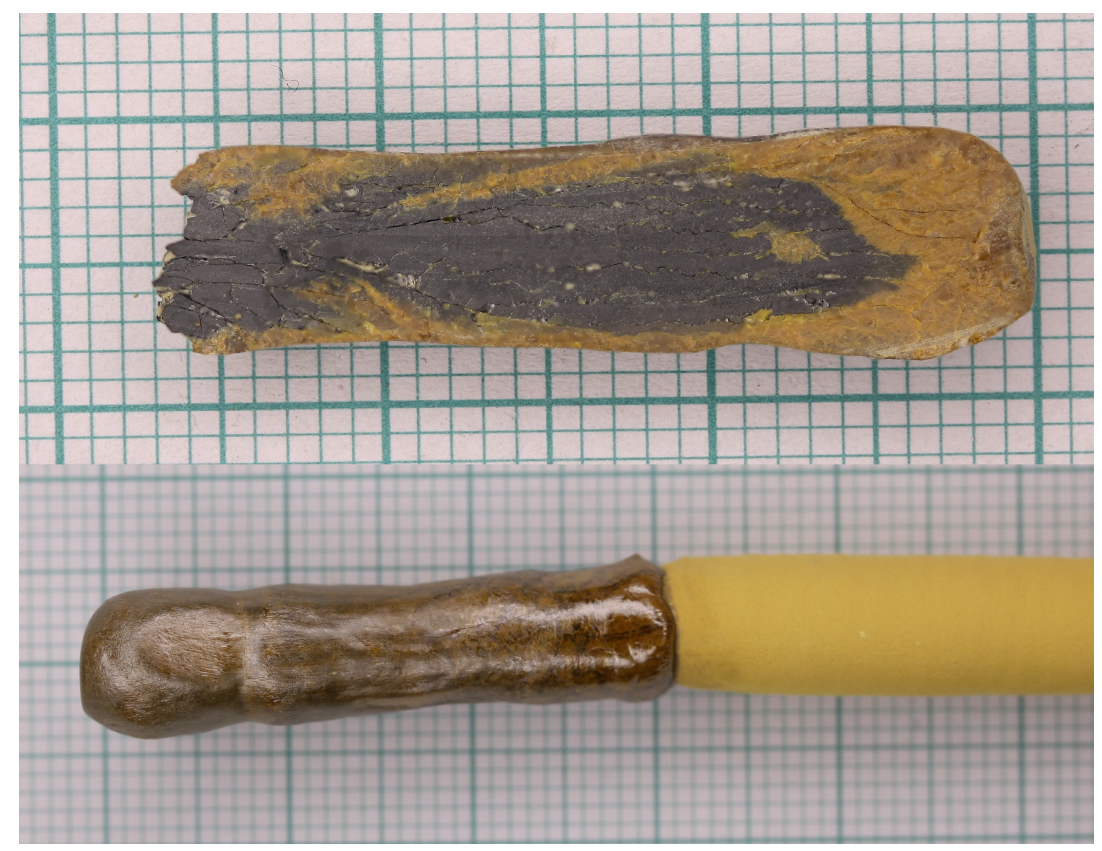

Figure 2. The result of growth 11 with $1.4 \%$ excess nickel oxide from stoichiometric. (a) The boule cut down the growth axis. There is a mixture of niobium-deficient $\beta-\mathrm{NiNb}_{2} \mathrm{O}_{6}$ (black) as well as polycrystalline columbite (yellow). (b) The as-grown boule.

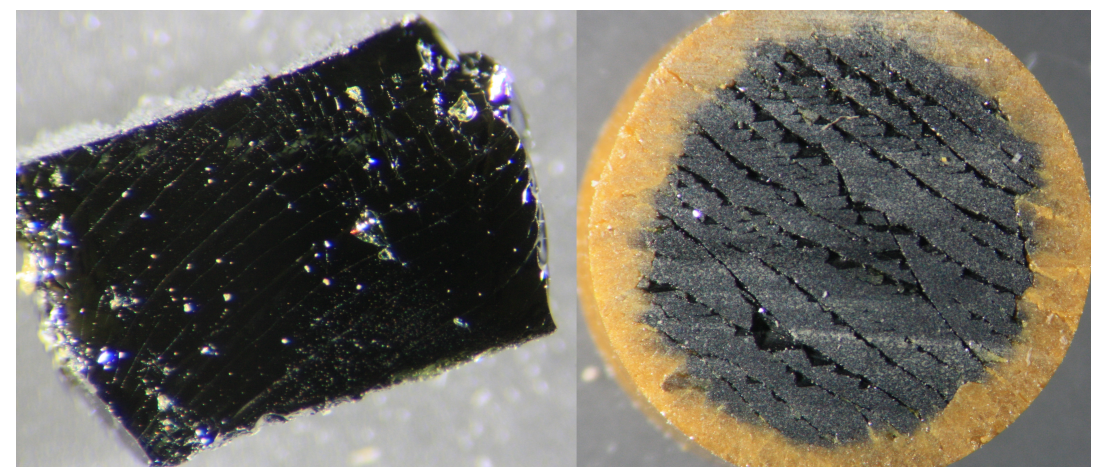

Figure 3. The result from growth 14 with $4 \%$ excess niobium oxide. (a) A cleaved plate of columbite $\mathrm{NiNb}_{2} \mathrm{O}_{6}$. The face shown is [110]. (b) A cross section cut of the material. Likely, the cracks formed on cooling due to a change in lattice parameters through a phase transition. A scalpel inserted into these cracks easily separated the grains. 


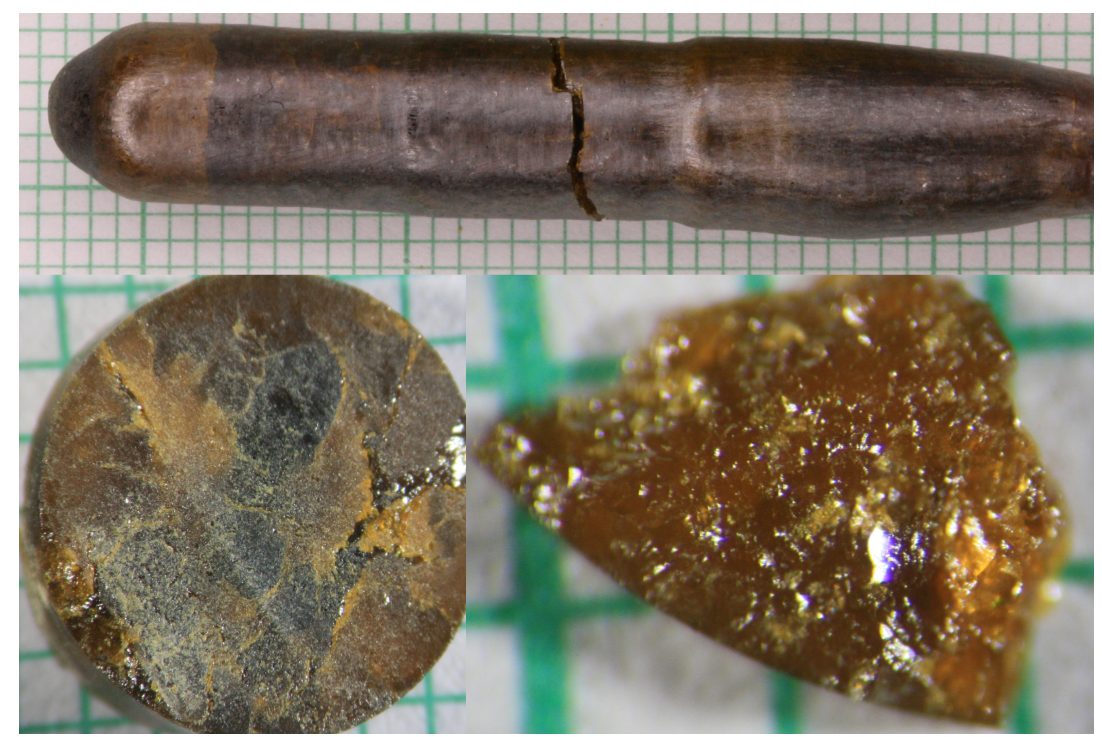

Figure 4. The result from growth 16 with $4 \%$ excess nickel oxide from stoichiometric. (a) The as grown boule, which cracked on removal from the image furnace. The dark, outer shell seemed to indicate that we had gotten closer to stabilizing the black phase. (b) A slice from inside the crystal, which shows definite mixture of phases despite the external picture. (c) A separated piece of columbite niobate crystal. Black pieces were also easily separated mechanically.

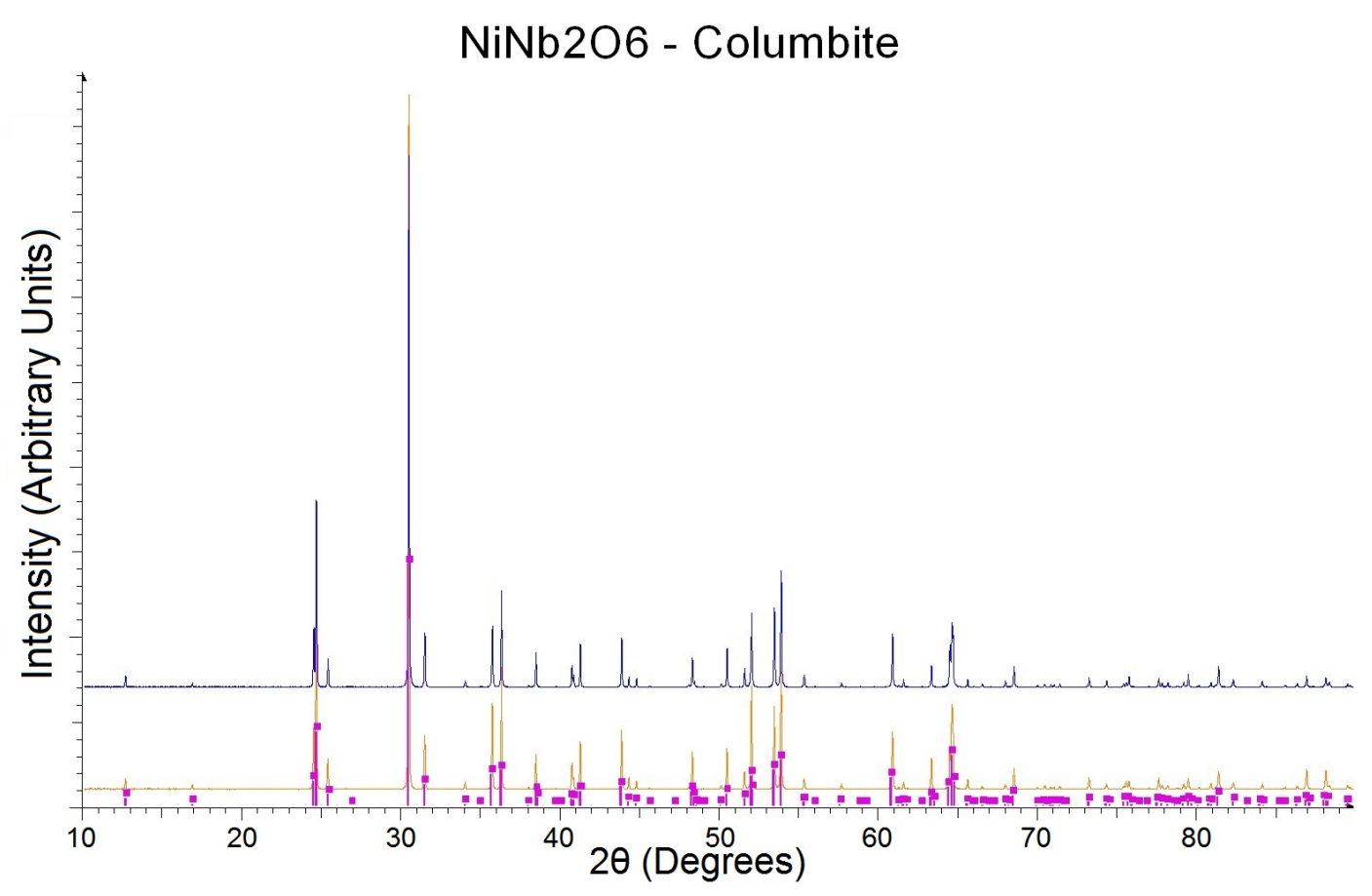

Figure 5. Powder x-ray diffraction from the black columbite phase single crystal (Top line, blue Growth 14), the yellow polycrystalline niobate (bottom line, yellow - From growth 12 , but essentially identical to all other growths) and a reference powder database (pattern 01-076-2354; $\mathrm{a}=14.032 \AA$, b $=5.687 \AA, c=5.033 \AA$ [19], magenta, peak intensity scaled arbitrarily.) 


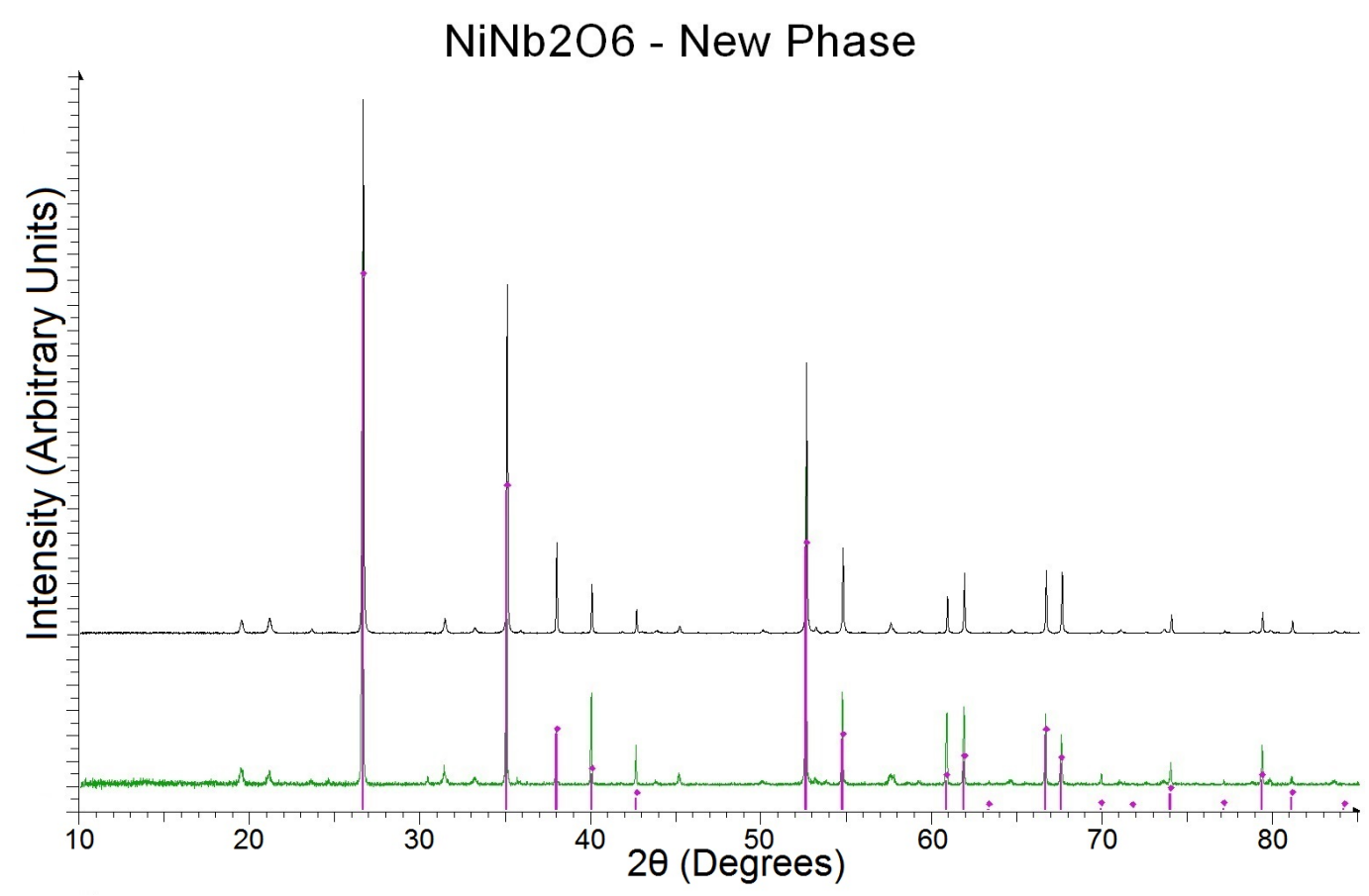

Figure 6. Powder x-ray diffraction from the black $\beta-\mathrm{NiNb}_{2} \mathrm{O}_{6}$ single crystal (Top line, black - Growth 15), the green single crystal (Bottom line, green - Growth 10), and a reference database orthorhombic cell, reported as tetragonal (pattern 01-076-2355; $a=4.71 \AA, c=3.038 \AA$, space group 136 [19] with scaled lattice parameters, magenta, peak intensity scaled arbitrarily)

result as niobium is not the magnetic species in this system, and a change like this would only lead to a small reduction in the nickel moment size due to the gain of a small percentage of extra electrons for charge balancing.

Using excess nickel oxide flux, we were able to significantly lower the growth temperature (by roughly 200 degrees). This allows us to avoid quenching and stabilized the columbite phase. Additionally, it allowed for more stable growth. This is to be expected as viscosity is inversely proportional to temperature. The nickel niobate in the columbite structure that were grown still had a thin layer of yellow polycrystalline material on the outside. The black internal material was identical to the polycrystalline shell, confirmed via powder XRD. With a lower temperature we obtained an external layer of polycrystalline material that could only be seen under magnification using the stereoscope; in essence we had eliminated the shell. The grown material was a mosaic of single crystals, both amber coloured and black, that were small (1-3 $\mathrm{mm}$ across).

\section{Materials and Methods}

The materials were prepared using $5 \mathrm{~N}$-purity niobium(V) oxide $\left(\mathrm{Nb}_{2} \mathrm{O}_{5}\right)$ and $4 \mathrm{~N}$-purity nickel(II) oxide (NiO). Each of these materials was separately placed in a ceramic crucible and heated to $725 \mathrm{~K}$ in air for 12 hours with a ramp rate of $100 \mathrm{~K}$ per hour to remove any moisture. Weighting and hand-mixing of the oxides either stoichiometrically or with various excesses of $\mathrm{NiO}$ or $\mathrm{Nb}_{2} \mathrm{O}_{5}$ was performed. The oxides were pre-reacted in ceramic crucibles at $1475 \mathrm{~K}$ for 48 hours with a ramp rate of $100 \mathrm{~K}$ per hour and were subjected to intermediate grinding.

The obtained materials were checked for complete reaction using a PANalytical X'Pert powder X-ray diffractometer on a low-background silicon disk. The patterns were checked against the standards for nickel oxides, niobium oxides and other nickel-niobium oxide compounds, such as $\mathrm{Ni}_{4} \mathrm{Nb}_{2} \mathrm{O}_{9}$ and the obtained materials were reground and re-heated if necessary. 
Once the powder was successfully synthesized, the materials were shaped into rods and placed in a hydrostatic press under a pressure of $50 \mathrm{MPa}$ for 15 minutes. The rods were removed from their forms, placed in a ceramic boat and then fired at $1475 \mathrm{~K}$ for 48 hours. Both seed and feed rods were made in this manner. The rods were approximately 6 to $7 \mathrm{~mm}$ in diameter and 8 to $11 \mathrm{~cm}$ in length.

The seed rod as hung on nickel wire in the OFZ furnaces. For the growth using a flux, a $5 \mathrm{~mm}$ thick $\mathrm{NiO}$ flux pellet was used.

The materials were heated in the OFZ furnace initially at a ramp rate of approximately $1.5 \%$ power per minute and, in all cases, the seed and the feed rotating at approximately $6 \mathrm{rpm}$ in the same direction. Once melting was evident, the rods were connected via a liquid bridge and counter-rotation was started. After a period of 1 to 2 minutes to stabilize, the growths were begun. Rotation was done in a counter-rotating fashion and speeds between 10 and 40 RPM were attempted, with 10 RPM on the top rod and $30 \mathrm{RPM}$ on the bottom rod being the most successful.

During the growth, significant changes were only made at the beginning of the growth unless otherwise needed. Initial increases in rotation speeds were done in 5 minute intervals, and in steps of no more than $5 \mathrm{rpm}$. Temperature adjustments were made as necessary, depending on the growth speed. As an example, an experiment done with between 5 and $7 \mathrm{~mm} / \mathrm{hr}$ growth speed required a delay of approximately 15 minutes to see changes unless exceptional circumstances required a manual adjustment.

When a growth was terminated, the rod separation was conducted by reducing lamp power at a rate of $1 \%$ per minute until the liquid zone was terminated. At this point the upper rod rotation and movement were stopped, the lower rotation speed was reduced in $5 \mathrm{rpm}$ steps and 10 second intervals to $6 \mathrm{rpm}$, the seed speed was set to zero and the cooling rate was set to $3 \%$ power per hour down to $25 \%$ lamp power, and $5 \%$ power per hour below that. For growths that were quenched, the procedure was modified to cool at 1\% lamp power per minute down to the appropriate lamp power.

Material assessment by powder XRD was performed. Pieces of crystal were either cut or mechanically separated, and then ground into a fine powder for $\mathrm{x}$-ray diffraction. Separated crystals were analyzed using a Laue diffractometer to determine growth direction. The original classification of $\beta-\mathrm{NiNb}_{2} \mathrm{O}_{6}$ was done using single crystal XRD and is reported in reference [24].

\section{Conclusions}

Through the processes described above, we have grown $5 \mathrm{~mm} \times 3 \mathrm{~mm} \times 1 \mathrm{~mm}$ sized crystals of pseudo-congruently melting (congruent composition different than stoichiometric composition) nickel niobate via the optical floating zone method. We found, separated and assessed a new phase in space group $86\left(\mathrm{P}_{2} / \mathrm{n}\right)$, confirmed by powder $\mathrm{x}$-ray diffraction. We have explored how the growth conditions are affected in the nickel-rich and niobium-rich cases, and how those conditions change the polymorph that is stabilized. In the nickel rich starting material we predominantly formed the columbite-structure (space group 60, $\mathrm{Pbcn}$ ) of nickel niobate as the excess $\mathrm{NiO}$ allowed us to work at a lower temperature. In the niobium rich material the growth resulted in the $\beta-\mathrm{NiNb}_{2} \mathrm{O}_{6}$ polymorph, which required a higher temperature and a thermal quench after growth to get the best quality single crystals. We are presently examining and comparing the magnetic properties of these different polymorphs.

Acknowledgements:The authors would like to thank Dr. J.E. Greedan and Dr. A. Dabkowski for useful discussions on experimental direction. Research at McMaster is supported by a NSERC grant from the Government of Canada. TJSM would like to acknowledge support in the form of an OGS grant from the Ontario Provincial Government.

Author Contributions: "T.M., G.L and H.D. conceived and designed the experiments; T.M., A.M. and H.D. performed the crystal growths; T.M. performed the x-ray scattering; T.M. analyzed the data; T.M. wrote the paper with input from all authors."

Conflicts of Interest: "The authors declare no conflict of interest. The founding sponsors had no role in the design of the study; in the collection, analyses, or interpretation of data; in the writing of the manuscript, and in the decision to publish the results". 


\section{Abbreviations}

The following abbreviations are used in this manuscript:

OFZ: Optical Floating Zone

QCP: Quantum Critical Point

NSERC: Natural Science and Engineering Research Council

OGS: Ontario Graduate Scholarship

rpm: revolutions per minute

XRD: X-Ray Diffraction

\section{References}

1. Dabkowska, H.A.; Dabkowski, A.B. Crystal Growth of Oxides by Optical Floating Zone Technique. In Springer Handbook of Crystal Growth; Dhanaraj, G., Byrappa, K., Prasad, V., and Dudley, M. Eds.; Springer Berlin Heidelberg: Berlin, Germany, 2010; pp. 367-391.

2. Kinross, A.W.; Fu, M.; Munsie, T.J.; Dabkowska, H.A.; Luke, G.M.; Sachdev, S.; and Imai, T. Evolution of Quantum Fluctuations Near the Quantum Critical Point of the Transverse Field Ising Chain System $\mathrm{CoNb}_{2} \mathrm{O}_{6}$.Phys. Rev. X 2014, 4, 031008.

3. Kobayashi, S.; Mitsuda, S.; Ishikawa, M.; Miyatani, K.; and Kohn, K. Three-Dimensional Magnetic Ordering in the Quasi- One-Dimensional Ising Magnet $\mathrm{CoNb}_{2} \mathrm{O}_{6}$ with Partially Released Geometrical Frustration. Phys. Rev. B 1999, 60, 3331.

4. Kunimoto, T.; Nagasaka, K.; Nojiri, H.; Luther, S.; Motokawa, M.; Ohta, H.; Goto, T.; Okubo, S.; and Kohn, K. Submillimeter Wave ESR Study of Magnetic Excitations in the Ising Ferromagnetic Chain $\mathrm{CoNb}_{2} \mathrm{O}_{6}$. J. Phys. Soc. Jpn. 1999, 68, 1703.

5. Coldea, R.; Tennant, D.A.; Wheeler, E.M.; Wawrzynska, E.; Prabhakaran, D.; Telling, M.; Habicht, K.; Smeibidl, P.; and Kiefer, K. Quantum Criticality in an Ising Chain: Experimental Evidence for Emergent $\mathrm{E}_{8}$ Symmetry. Science 2010, 327, 177.

6. Morris, C.M.; Aguilar, R.V.; Ghosh, A.; Koohpayeh, S.M.; Krizan, J.; Cava, R.J.; Tchernyshyov, O.; McQueen, T.M.; and Armitage, N.P. Hierarchy of Bound States in the One-Dimensional Ferromagnetic Ising Chain $\mathrm{CoNb}_{2} \mathrm{O}_{6}$ Investigated by High-Resolution Time-Domain Terahertz Spectroscopy.Phys. Rev. Lett. 2014, 112, 137403.

7. Hertz, J.A.; Quantum Critical Phenomena.Phys. Rev. B 1976, 14, 1165.

8. Chakravarty, S.; Halperin, B.I.; and Nelson, D.R. Two-Dimensional Quantum Heisenberg Antiferromagnet at Low Temperatures.Phys. Rev. B 1989, 39, 2344.

9. Millis, A.J.; Effect of a Nonzero Temperature on Quantum Critical Points in Itinerant Fermion Systems.Phys. Rev. B 1993, 48, 7183.

10. Chubukov, A.V.; Sachdev, S.; and Ye, J. Theory of Two-Dimensional Quantum Heisenberg Antiferromagnets with a Nearly Critical Ground State.Phys. Rev. B 1994, 49, 11919.

11. Goldman, A.M.; and Markovic, N. Superconductor-Insulator Transitions in the Two-Dimensional Limit.Phys. Today 1998, 51, 39.

12. Sachdev, S. Quantum Magnetism and Criticality. Nat. Phys 2008, 4, 173.

13. Gegenwart, P.; Si, Q.; and Steglich, F. Quantum Criticality in Heavy-Fermion MetalsNat. Phys 2008, 4, 186.

14. Sachdev, S.; Quantum Phase Transitions; 2nd Ed.; Cambridge University Press: Cambridge, England, 2011;

15. Sachdev, S.; and Keimer, B. Quantum CriticalityPhys. Today 2011, 64, 29.

16. Scharf, W.; Weitzel, H.; Yaeger, I.; Maartense, I.; and Wanklyn, B.M. Magnetic Structures of $\mathrm{CoNb}_{2} \mathrm{O}_{6}$.J. Magn. Magn. Mater. 1979, 13, 121.

17. Weitzel, H. Kristallstrukturverfeinerung von Wolframiten und Columbiten.Z. Kristal. 1976, 144, 238.

18. Sarvezuk, P.W.C.; Kinast, E.J;; Colin, C.V.; Gusmão, M.A.;Ǐ da Cunha, J.B.M.; and Isnard, O. New investigation of the magnetic structure of $\mathrm{CoNb}_{2} \mathrm{O}_{6}$ columbite.J. App. Phys. 2011, 109, $07 \mathrm{E} 160$.

19. Wichmann, R.; and Mueller-Buschbaum, H. Synthese und Untersuchung von Ni Nb2 O6-Einkristallen mit Columbit - und Rutilstruktur.Z. Anorgan. All. Chem. 1983, 503, 101. 
233

234

235

236

237

238

239

240

241

242

243

244

245

20. Prabhakaran, D.; Wondre, F.R.; and Boothroyd, A.T. Preparation of large single crystals of $\mathrm{ANb}_{2} \mathrm{O}_{6}(\mathrm{~A}=\mathrm{Ni}$, Co, Fe, Mn) by the floating-zone method.J. Crys. Growth 2003, 1-2, 72.

21. Burdese, A.; Lucco-Borlera, M.; and Rolando, P. Phase Diagram of $\mathrm{Nb}_{2} \mathrm{O}_{5}-\mathrm{NiO}$. Ric. Sci., Rend. 1964, A7, 454.

22. Yaeger, I.; Morrish, A.H.; and Wanklyn, B.M. Magnetization studies in transition-metal niobates: I. $\mathrm{NiNb}_{2} \mathrm{O}_{6}$ Phys. Rev. B. 1977, 3, 1465.

23. Heid, C.; Weitzel, H.; Bourdarot, F.; Calemczuk, R.; Vogt, T.; and Fuess, H. Magnetism in FeNb $\mathrm{O}_{6}$ and $\mathrm{NiNb}_{2} \mathrm{O}_{6}$ J. Cond. Mat. 1996, 49, 10609.

24. Munsie, T.J.S.; Millington, A.; Dube, P.A.; Dabkowska, H.A.; Britten, J.; Luke, G.M.; and Greedan, J.E. Crystal growth and magnetic characterization of a tetragonal polymorph of $\mathrm{NiNb}_{2} \mathrm{O}_{6}$.J. Solid State Chem. 2015, 236, 19.

25. Beck, H.P. A study on $\mathrm{AB}_{2} \mathrm{O}_{6}$ compounds, Part II: the branches of the hcp family.Z. Kristallogr. 2012, 227, 843. 